\title{
Isolation of protein $\mathbf{N}$-terminal peptides by charge-mediated position- selective enrichment using strong cation exchange chromatography
}

Chih-Hsiang Chang ${ }^{1}$, Hsin-Yi Chang ${ }^{1}$, Juri Rappsilber ${ }^{2,3}$ and Yasushi Ishihama ${ }^{1 *}$

${ }^{1}$ Graduate School of Pharmaceutical Sciences, Kyoto University, Kyoto 606-8501, Japan.

${ }^{2}$ Bioanalytics, Institute of Biotechnology, Technische Universität Berlin, 13355 Berlin, Germany.

${ }^{3}$ Wellcome Centre for Cell Biology, School of Biological Sciences, University of Edinburgh, Edinburgh EH9 3BF, United Kingdom.

Running title: Isolation of protein N-terminal peptides 


\section{Abbreviations}

CHAMP, charge-mediated position-selective enrichment

SCX, strong cation exchange chromatography

MS, mass spectrometry

COFRADIC, combined fractional diagonal chromatography

TAILS, terminal amine isotopic labeling of substrates

ChaFRADIC, charge-based fractional diagonal chromatography

HYTANE, hydrophobic tagging-assisted N-termini enrichment

Tris-HCl, 2-amino-2-(hydroxymethyl)-1,3-propanediol hydrochloride

SDC, sodium deoxycholate

SLS, sodium N-lauroylsarcosinate

TCEP, tris(2-carboxyethyl)phosphine

CAA, 2-chloroacetamide

ACN, acetonitrile,

TFA, trifluoroacetic acid

PTS, phase-transfer surfactants

StageTip, stop and go extraction tip 


\begin{abstract}
We developed a simple and rapid method to enrich protein N-terminal peptides, in which the protease TrypN is first employed to generate protein N-terminal peptides without Lys or Arg and internal peptides with two positive charges at their N-termini, and then the Nterminal peptides are separated from the internal peptides by means of CHArge-Mediated Position-selective (CHAMP) enrichment using strong cation exchange (SCX) chromatography. This CHAMP-SCX approach was applied to $20 \mu \mathrm{g}$ of human HEK293T cell lysate proteins to profile the N-terminal proteome. On average, 1,550 acetylated and 200 unmodified protein N-terminal peptides were successfully identified in a single LC/MS/MS run with less than $3 \%$ contamination with internal peptides, even when we accepted only canonical protein N-termini registered in the Swiss-Prot database. Since this method involves only two steps, protein digestion and chromatographic separation, without the need for tedious chemical reactions, it should be useful for comprehensive profiling of protein $\mathrm{N}$ termini, including proteoforms with neo-N-termini.
\end{abstract}




\section{INTRODUCTION}

Characterizing protein $\mathrm{N}$-termini is essential to understand how the entire proteome is generated through biological processes such as translational initiation (1-3), posttranslational modifications $(4,5)$ and proteolytic cleavages $(6,7)$. In order to perform $\mathrm{N}$ terminomics using mass spectrometry (MS), peptides derived from protein N-termini must be selectively enriched, and many methods have been developed for this purpose $(8,9)$. Some of them use “positive selection” approaches in which chemically labeled protein N-terminal peptides are enriched by affinity purification $(6,10)$. However, these approaches are difficult to apply to protein $\mathrm{N}$-termini with modifications. In contrast, “negative selection” approaches to isolate protein N-terminal peptides by depleting internal peptides have been used to comprehensively identify protein N-terminal peptides, including $\mathrm{N}$-terminal modifications such as methylation, acetylation, and lipidation $(11,12)$. Gevaert et al. pioneered combined fractional diagonal chromatography (COFRADIC) (13), and this was followed by other negative selection approaches such as terminal amine isotopic labeling of substrates (TAILS) (14), the variant of COFRADIC called charge-based fractional diagonal chromatography (ChaFRADIC) (15), and hydrophobic tagging-assisted N-termini enrichment (HYTANE) (16). All of them require blocking of the primary amines at the protein level and depletion of digested internal peptides by means of chemical tagging-based separation. Thus, relatively large amounts of samples ( $\sim 5$ to $10 \mathrm{mg}$ ) are generally required to increase the identification number of protein N-terminal peptides. This limits the usefulness of these approaches in the case of hard-to-obtain biological samples $(17,18)$. Furthermore, limitations in the efficiency and specificity of the chemical derivatizations compromise the confidence of peptide 
identification. Therefore, a simple and sensitive approach to enrich protein N-terminal peptides is still needed for MS-based proteomics.

Strong cation exchange (SCX) chromatography, employing Coulombic interactions to separate peptides based on their positive charges, has been widely applied for deep proteomic profiling $(19,20)$. In SCX separation of tryptic peptides, abundant acetylated protein Nterminal peptides are eluted first. Peptides with 1+ charge such as monophosphorylated peptides, N-pyroglutamated peptides and protein C-terminal peptides are then simultaneously eluted, followed by peptides with +2 or more net charge, such as unmodified protein N-terminal peptides, internal peptides and peptides containing missed cleavages (21, 22). Thus, it is impossible to isolate protein $\mathrm{N}$-terminal peptides from tryptic peptides by SCX chromatography. To overcome this issue, we focused on TrypN (23), also known as LysargiNase, a metalloprotease that cleaves peptide chains mainly at the N-terminal side of Lys/Arg even in the case of Pro-Lys and Pro-Arg bonds, generating peptides with N-terminal Lys/Arg and yielding protein N-terminal peptides that do not contain Lys/Arg. Unlike other LysargiNases such as ulilysin $(24,25)$ and mirolysin (26), which preferentially cleave the Nterminal side of either Lys or Arg, TrypN cleaves the N-terminal side of Lys and Arg equally at pH 6 8. Moreover, the peptide identification performance for $\mathrm{N}$-terminal Lys/Arg peptides is comparable to that for tryptic peptides (27).

In this study, we developed a new method to enrich protein $\mathrm{N}$-terminal peptides without the need for chemical derivatization or complex procedures, taking advantage of the combination of proteinase TrypN-mediated protein cleavage and SCX separation of Nterminal peptides based on the charge difference at the $\mathrm{N}$-termini of protein $\mathrm{N}$-terminal peptides and internal peptides. We call this method CHArge-Mediated Position-selective 
(CHAMP) enrichment using strong cation exchange (SCX) chromatography (CHAMP-SCX).

We show that this rapid and simple approach to enrich protein N-terminal peptides enables comprehensive, high-throughput analysis of the human N-terminal proteome.

\section{MATERIALS AND METHODS}

\section{Materials}

Ammonium bicarbonate, 2-amino-2-(hydroxymethyl)-1,3-propanediol hydrochloride (Tris-HCl), sodium deoxycholate (SDC), sodium N-lauroylsarcosinate (SLS), ammonium bicarbonate, tris(2-carboxyethyl)phosphine (TCEP), 2-chloroacetamide (CAA), calcium chloride, ethyl acetate, acetonitrile (ACN), acetic acid, trifluoroacetic acid (TFA) and other chemicals were purchased from Fujifilm Wako (Osaka, Japan). Modified trypsin was from Promega (Madison, MA). TrypN was from Protifi (Huntington, NY). Styrene divinylbenzene (SDB-XC) Empore ${ }^{\mathrm{TM}}$ disk and strong cation exchange (Cation-SR) Empore $^{\mathrm{TM}}$ disks were purchased from GL Sciences (Tokyo, Japan). Water was purified by a Millipore Milli-Q system (Bedfold, MA, USA).

\section{Escherichia coli cell culture and protein extraction}

Escherichia coli K-12 BW25113 was grown to mid-log phase in LB broth with vigorous shaking at $37^{\circ} \mathrm{C}$. Cells were collected by centrifugation and resuspended in buffer containing protease inhibitors (Sigma), 12 mM SDC, 12 mM SLS, 10 mM TCEP, 40 mM CAA in 100 $\mathrm{mM}$ Tris buffer ( $\mathrm{pH}$ 8.5). The lysate was vortexed and sonicated on ice for $20 \mathrm{~min}$. The concentration of protein crude extract was determined by means of bicinchoninic acid (BCA) protein assay (Thermofisher Scientific, Rockford, IL, USA). 


\section{HEK293T cell culture and protein extraction}

HEK293T (human embryonic kidney) cells, cultured to $80 \%$ confluence in $10-\mathrm{cm}$ diameter dishes, were harvested in lysis buffer containing protease inhibitors (Sigma), 12 mM SDC, 12 mM SLS, 10 mM TCEP, 40 mM CAA in 100 mM Tris buffer (pH 8.5). The lysate was vortexed and sonicated on ice for $20 \mathrm{~min}$. The final protein concentration of the sample was determined using the BCA protein assay.

\section{Protein Digestion}

For TrypN digestion, the quantified protein solution was diluted 10 -fold with $10 \mathrm{mM}$ $\mathrm{CaCl}_{2}$ and digested with TrypN (1: $\left.50 \mathrm{w} / \mathrm{w}\right)$ overnight at $37{ }^{\circ} \mathrm{C}$. Note that TrypN can be replaced with LysargiNase (Merck Millipore, Darmstadt, Germany). In the case of tryptic digestion, the protein solution was digested with Lys-C $(1: 50 \mathrm{w} / \mathrm{w})$ for $3 \mathrm{~h}$ at $37^{\circ} \mathrm{C}$, followed by 5 -fold dilution with $50 \mathrm{mM}$ ammonium bicarbonate and trypsin digestion (1:50 w/w) overnight at $37^{\circ} \mathrm{C}$. After enzymatic digestion, an equal volume of ethyl acetate was added to each sample solution, and the mixture was acidified with $0.5 \%$ trifluoroacetic acid (final concentration) according to the PTS protocol reported previously (28). The resulting mixture was shaken for 1 min and centrifuged at 15,700 g for 2 min to separate the ethyl acetate layer. The aqueous layer was collected and desalted by using StageTips with SDB-XC disk membranes (29). The proteolytic peptides were quantified by LC-UV at $214 \mathrm{~nm}$ with standard BSA peptides and kept in $80 \%$ ACN and $0.5 \%$ TFA at $-20{ }^{\circ} \mathrm{C}$ until use.

\section{Peptide fractionation by SCX HPLC}


SCX chromatography was performed using a Prominence HPLC system (Shimadzu, Kyoto, Japan) with a BioIEX SCX column $(250 \mathrm{~mm} \times 4.6 \mathrm{~mm}$ inner diameter, $5 \mu \mathrm{m}$ nonporous beads made of poly(styrene-divinylbenzene) modified with sulfonate groups (Agilent, Santa Clara, CA, USA).

For examination of the SCX separation characteristics, $75 \mu \mathrm{g}$ each of trypsin- and TrypNdigested HEK293T peptides were mixed and directly loaded onto the SCX column at 0.8 $\mathrm{mL} / \mathrm{min}$. A mixture of $5 \mathrm{mM}$ potassium phosphate (pH 3.0) and ACN (7:3) was used as SCX buffer $\mathrm{A}$, and a mixture of $500 \mathrm{mM}$ potassium phosphate (pH 3.0) and ACN (7:3) was used as SCX buffer B. Gradient elution was performed as follows: 0\% B for 5 min, 0-10\% in 20 min, $10-50 \%$ in 10 min, 50-100\% in 5 min and 100\% B for 4 min. Fractions were manually collected at one min intervals for $45 \mathrm{~min}$. After evaporation of the solvent in a SpeedVac SPD121P (Thermofisher Scientific), fractionated peptides were resuspended in $50 \mu \mathrm{L}$ of 0.1\% TFA and desalted by using StageTips with SDB-XC disk membranes. One-fourth of each fraction was analyzed by nanoLC/MS/MS using a TripleTOF 5600 (SCIEX, Foster City, CA, USA) as described below.

For gradient SCX fractionation of TrypN-digested HEK293T peptides, $80 \mu \mathrm{g}$ of digested peptides were analyzed using the SCX HPLC system described above. A mixture of $7.5 \mathrm{mM}$ potassium phosphate (pH 2.6) and ACN (7:3) was used as SCX buffer A, and $350 \mathrm{mM} \mathrm{KCl}$ was added to buffer A for SCX buffer B. Gradient elution was performed as follows: 0.5\% B for 15 min, 0.5-1\% B in 10 min, 1-4\% B in 10 min, 4-10\% B in 3 min, 10-100\% B in 3 min, and 100\% B for 5 min. Fractions were manually collected at one min intervals for 50 min. After evaporation of the solvent in a SpeedVac, fractionated peptides were resuspended in $50 \mu \mathrm{L}$ of $0.1 \%$ TFA and desalted by using StageTips with SDB-XC disk membranes. One- 
fourth of each fraction for Fr.1-43 and one-tenth of each fraction for Fr.44-50 were analyzed by nanoLC/MS/MS using an Orbitrap Fusion Lumos mass spectrometer (Thermofisher Scientific) as described below.

\section{Enrichment of protein N-terminal peptides by CHAMP-SCX}

Enrichment of protein N-terminal peptides from $30 \mu \mathrm{g}$ of TrypN-digested E. coli peptides was performed using the SCX HPLC system under the following isocratic conditions: SCX buffer A was a mixture of 7.5 mM potassium phosphate solution ( $\mathrm{pH} 2.2$ ) containing 10, 12.5 or $15 \mathrm{mM} \mathrm{KCl}$ and ACN (7:3), and buffer B was a mixture of $7.5 \mathrm{mM}$ potassium phosphate solution (pH 2.2) containing $500 \mathrm{mM} \mathrm{KCl}$ and ACN (7:3). Isocratic elution was performed with $100 \%$ A for 30 min and then the system was washed with $100 \%$ B. The collected eluates were dried in a SpeedVac and the residue was resuspended in $50 \mu \mathrm{L}$ of $0.1 \%$ TFA and desalted on StageTips with SDB-XC disk membranes. Two-thirds of the enriched peptides were analyzed by nanoLC/MS/MS using the Orbitrap Fusion Lumos.

To isolate protein N-terminal peptides from TrypN-digested HEK293T peptides, the digested peptides (80 $\mu$ g) were analyzed by the SCX HPLC system under isocratic conditions, eluting with a mixture of $7.5 \mathrm{mM}$ potassium phosphate solution (pH 2.2) containing $10 \mathrm{mM}$ $\mathrm{KCl}$ and $\mathrm{ACN}$ (7:3) for 30 min to collect the desired fraction. After evaporation of the solvent in a SpeedVac, enriched peptides were resuspended in $50 \mu \mathrm{L}$ of $0.1 \%$ TFA and desalted using StageTips with SDB-XC disk membranes. We analyzed one-fourth of the enriched peptides by nanoLC/MS/MS in triplicate using the Orbitrap Fusion Lumos.

\section{NanoLC/MS/MS analysis}


NanoLC/MS/MS analyses were performed on a TripleTOF 5600 mass spectrometer or an Orbitrap Fusion LUMOS mass spectrometer, connected to a Thermo Ultimate 3000 pump and an HTC-PAL autosampler (CTC Analytics, Zwingen, Switzerland). Peptides were separated on self-pulled needle columns $(150 \mathrm{~mm}$ length $\times 100 \mu \mathrm{m}$ ID, $6 \mu \mathrm{m}$ opening $)$ packed with Reprosil-C18 $3 \mu \mathrm{m}$ reversed-phase material (Dr. Maisch, Ammerbuch, Germany). The injection volume was $5 \mu \mathrm{L}$ and the flow rate was $500 \mathrm{~nL} / \mathrm{min}$. The mobile phases were (A) 0.5\% acetic acid and (B) 0.5\% acetic acid and 80\% ACN. For TripleTOF 5600 analysis, gradient elution was performed as follows: $12-40 \% \mathrm{~B}$ in $20 \mathrm{~min}, 45-100 \%$ B in $1 \mathrm{~min}, 100 \%$ B for 5 min. For Orbitrap analysis, gradient elution of fractionated samples was performed as follows: $12-40 \% \mathrm{~B}$ in $15 \mathrm{~min}, 40-100 \% \mathrm{~B}$ in $1 \mathrm{~min}, 100 \% \mathrm{~B}$ for $5 \mathrm{~min}$. For protein $\mathrm{N}-$ terminal peptide-enriched samples, gradient elution was performed as follows: $10-40 \% \mathrm{~B}$ in $100 \mathrm{~min}, 40-100 \%$ B in $10 \mathrm{~min}, 100 \%$ B for $10 \mathrm{~min}$. Spray voltages of $2300 \mathrm{~V}$ in the TripleTOF 5600 system and $2400 \mathrm{~V}$ in the Orbitrap system were applied. The mass scan range of the TripleTOF 5600 system was $m / z$ 300-1500, and the top ten precursor ions were selected in each MS scan for subsequent MS/MS scans. The mass scan range for the Orbitrap system was $m / z$ 300-1500, with an automatic gain control value of $1.00 \mathrm{e}+06$, a maximum injection time of $50 \mathrm{~ms}$ and detection at a mass resolution of 60,000 at $\mathrm{m} / \mathrm{z} 200$ in the orbitrap analyzer. The top ten precursor ions were selected in each MS scan for subsequent MS/MS scans with an automatic gain control value of 5.00e +04 and a maximum injection time of 300 ms. Dynamic exclusion was set for 25 s with a 10 ppm gate. The normalized HCD was set to be 30, with detection at a mass resolution of 15,000 at $\mathrm{m} / \mathrm{z} 200$ in the Orbitrap analyzer. A lock mass (445.1200025) function was used to obtain constant mass accuracy during the gradient. 


\section{Proteomics Data Processing}

Two peak lists in “.mgf” and “.apl” formats were generated from the MS/MS spectra by MaxQuant (30). The peptides and proteins were identified by Mascot v2.6.1 (Matrix Science, London, U.K.) against the Swiss-Prot database (version 2017_4, 20,199 sequences) or the E. coli K-12 MG1665 protein sequence database (31) with a precursor mass tolerance of 20 ppm (TripleTOF 5600) or $10 \mathrm{ppm}$ (Orbitrap), a fragment ion mass tolerance of $0.1 \mathrm{Da}$ (TripleTOF 5600) or 20 ppm (Orbitrap), TrypN/trypsin specificity allowing for up to 4 missed cleavages for TrypN/trypsin mixed proteolytic peptides and strict TrypN specificity allowing for up to 2 missed cleavages for TrypN-digested peptides. Carbamidomethylation of cysteine was set as a fixed modification, and methionine oxidation and protein N-terminal acetylation were allowed as variable modifications. False discovery rates at a peptide level of less than $1 \%$ were applied for peptide identification based on a target-decoy approach.

\section{RESULTS AND DISCUSSION}

\section{Charge-mediated position-selective enrichment in SCX chromatography}

Proteolysis with TrypN yields peptides with at least a +2 charge with Lys or Arg and an $\alpha$-amino group at the $\mathrm{N}$-terminus. On the other hand, peptides derived from protein $\mathrm{N}$-termini have neither Lys nor Arg and are highly acetylated at the N-terminus, so that most of them have a 0 or +1 charge, and only His-containing peptides with an unmodified $\mathrm{N}$-terminus have $\mathrm{a}+2$ charge. In this study, we focused on the fact that SCX chromatography at low $\mathrm{pH}$ can separate peptides based on the number of positive charges, and we attempted to separate protein N-terminal peptides from internal peptides among TrypN-digested peptides. 
We first examined the number of missed cleavages in TrypN digestion. When digestion was performed in $0.1 \%$ RapiGest according to the manufacturer's protocol, the missed cleavage rate (the content of peptides with two or more missed cleavage sites) was $14 \%$, almost equal to the value in the condition without addition of RapiGest (16\%). On the other hand, when 1\% SDC was added instead of RapiGest, the missed cleavage rate was dramatically reduced to 5.8\%. Thus, TrypN digestion was performed according to the PTS protocol (28) in this study.

Next, keeping in mind the need to separate protein N-terminal-derived peptides with His residues and unmodified N-termini from TrypN-digested internal peptides, we investigated whether the peptides could be separated based on the position of the positive charge, in addition to the number of positive charges, by SCX chromatography. Studies with proteases that cleave either Lys/Arg, such as Lys-C and Lys-N or trypsin and TrypN, have indicated that the position of the positive charge affects the outcome in shotgun proteomics $(25,32)$. For example, it has been reported that peptides with N-terminal Lys or Lys/Arg are more strongly retained than peptides with a C-terminal Lys or Lys/Arg in reversed-phase LC (27). To determine how the Lys/Arg position of peptides affects their retention behavior in SCX chromatography at low $\mathrm{pH}$, we examined a mixture of TrypN- and trypsin-digested peptides using the SCX HPLC system, followed by nanoLC/MS/MS. The 19,853 unique tryptic peptides generally showed weaker retention than the 11,334 unique TrypN peptides with the same charge states (Supplementary Fig. S1). To characterize the SCX elution profiles in more detail, we compared the retention time in SCX HPLC for approximately 4,000 peptide pairs having sequences that differ only in the presence or absence of terminal Lys/Arg (Figure 1A). As expected, a strong retention shift was observed for TrypN-digested peptides. This would 
be because the TrypN peptides are strongly positively charged at the $\mathrm{N}$-terminus, due to the $\alpha$-amino group of the $\mathrm{N}$-terminal Lys/Arg and the side chain $\varepsilon$-amino or guanidino group, whereas the positive charge of the C-terminal Lys/Arg of trypsin peptides was partially neutralized by the $\alpha$-carboxy group (Fig. 1B). Gauci et al. reported that Lys-N-digested phosphopeptides with two basic moieties in close proximity tend to be more strongly retained on an SCX column than tryptic phosphopeptides (22). Gussakovsky et al. reported a retention model for predicting the retention times in SCX chromatography of tryptic peptides, in which the position-dependent coefficient of basic amino acids is higher near the N-terminus (33). We also found that the TrypN-digested peptides eluted in a narrower SCX fraction range than the tryptic peptides (Fig. S1). This may be due to the fact that the N-terminal positive charge of the tryptic peptides differs depending upon the amino acid involved, whereas the $\mathrm{N}$ terminal amino acid of the TrypN-digested peptide is always Lys or Arg. To our knowledge, the present work is the first to show that the position of basic residues in the same sequence reliably affects the electrostatic interaction with the SCX stationary phase using thousands of identical sequence pairs, and we named this approach charge-mediated position-selective SCX (CHAMP-SCX) enrichment.

\section{CHAMP-SCX separation of TrypN-digested HEK293T peptides}

The HPLC system used in this study was equipped with a nonporous hydrophilic SCX column having a separation efficiency equivalent to that of a typical reversed-phase column (the peak width at half height was $12.4 \pm 4.2$ seconds and the peak capacity was 122 ; see Supplementary Fig S2). As already shown in Supplementary Figure S1, this SCX HPLC system was able to separate TrypN-digested peptides with +1 and +2 charges from each other. 
Comprehensive SCX fractionation of TrypN-digested peptides derived from HEK293T cells was performed with a $\mathrm{KCl}$ salt gradient elution at $\mathrm{pH}$ 2.6, and peptide identification for each fraction was performed by nanoLC/MS/MS. As shown in Figure 2A, nearly all of the protein N-terminal-derived peptides were clearly separated from the internal peptides, regardless of whether their N-termini were acetylated or not. The fractions from 2 to $11 \mathrm{~min}$ contained mainly 0 and +1 peptides, including 2,207acetylated protein N-terminal peptides, 345 Hiscontaining acetylated N-terminal peptides, and 262 unmodified N-terminal peptides. The 1218 min fractions contained +2 peptides, i.e., unmodified protein N-terminal peptides containing one His, Lys or Arg and acetylated protein N-terminal peptides containing two basic amino acids. The next fractions from 19 to 30 min also contained +2 peptides, but most of them were internal peptides based on the CHAMP effect, i.e., retention was stronger due to the high density of positive charge at the N-terminus of the peptides (Fig. 1B). Thus, the protein N-terminal peptides can be easily isolated. Peptides with a charge greater than 2+ were sequentially eluted in the fractions after $31 \mathrm{~min}$. These included protein N-terminal peptides containing missed cleavage sites, but their number was small due to the high efficiency of TrypN digestion by the PTS method. Up to $90 \%$ of non-redundant protein Nterminal peptides could be recovered in fractions up to 18 min by CHAMP-SCX separation (Supplementary Figure S3), demonstrating that the combination of TrypN digestion and CHAMP-SCX enables simple and rapid protein N-terminal peptide enrichment. In addition, unlike trypsin, which is unable to cleave Lys-Pro and Arg-Pro bonds, TrypN can cleave ProLys and Pro-Arg bonds, generating protein N-terminal peptides with Pro at the C-termini, and thus improving the coverage in $\mathrm{N}$-terminomics. 


\section{Optimization of CHAMP-SCX separation using TrypN-digested E. coli peptides}

To optimize the elution conditions for CHAMP separation, we employed E. coli TrypNdigested peptides. Because bacterial proteins have less $\mathrm{N}$-terminal modification than mammalian proteins, the bacterial sample was considered preferable to optimize the conditions for separating the protein N-terminal peptides with $2+$ charge (peptides with an unmodified N-terminus and one His residue) from the internal peptides. Three SCX buffers with different $\mathrm{KCl}$ concentrations were used for isocratic elution for $30 \mathrm{~min}$, and the enrichment efficiencies for protein N-terminal peptides were compared. An enrichment specificity of more than 97\% was obtained with $10 \mathrm{mM} \mathrm{KCl}$ (Table 1). When buffers with higher $\mathrm{KCl}$ concentrations were used, more internal peptides were identified (Figure 2B). In the case of $10 \mathrm{mM}$ KCI buffer, we identified 53 His-containing protein N-terminal peptides out of 270 non-redundant protein N-terminal peptides without missed cleavage from $20 \mu \mathrm{g}$ of E. coli lysate (19.6\%, Figure 2C). Among in-silico TrypN-digested peptides from the E. coli proteome, $20 \%$ of the protein $\mathrm{N}$-terminal peptides contain one His, suggesting that our enrichment conditions have no bias in identifying His-containing protein $\mathrm{N}$-terminal peptides. In other words, CHAMP-SCX chromatography was able to separate the protein N-terminal peptides from the internal peptides even in the most difficult cases where the unmodified protein N-terminal peptides contain an additional basic amino acid, such as His, Lys or Arg.

\section{HEK293T protein N-terminal peptide enrichment by CHAMP-SCX}

The N-terminal peptides of His-containing proteins could be successfully separated from the internal peptides of human and bacterial samples by means of CHAMP-SCX under optimized elution conditions. To validate the applicability of this method to large-scale N- 
terminal proteomics, we performed triplicate analyses using HEK293T cells, which have been widely used in N-terminal proteomics $(17,18)$. Triplicate SCX fractionations using 10 mM KCl isocratic elution were done for TrypN-digested HEK293T peptides (80 $\mu \mathrm{g}$ each time), and we subjected one-fourth of the isolated peptides to nanoLC/MS/MS in triplicate (9 runs in total). Default parameters, such as the Swiss-Prot human protein sequence database, specific TrypN cleavage, and minimum peptide length of 7 amino acids, were applied for peptide identification by database search (Figure 3A). The results are shown in Figure 3B and Table 2. High correlations of peak areas of identified peptides were observed for intraand interday preparation samples, $\left(\mathrm{R}^{2}=0.95\right.$ and $\mathrm{R}^{2}=0.75,0.80$, respectively). On average, we identified 1,550 unique acetylated and 200 unmodified protein N-terminal peptides from $20 \mu \mathrm{g}$ of TrypN-digested HEK293T peptides in a single LC/MS/MS analysis. Contamination by internal peptides amounted to only $3 \%$ and $9 \%$ in peptide peak area and peptide number, respectively (Figure 3C, Table 2). Protein N-terminal peptides with missed cleavage were also enriched in the same elution, and 850 ( 50\%) miscleaved unique N-terminal peptides were identified on average, improving the coverage of the N-terminome. We identified 1,640 acetylated, 106 partially acetylated and 167 unmodified non-redundant protein N-termini. Note that 1,600 additional neo-N-terminal peptides were identified when semi-specific cleavage at the $\mathrm{N}$-terminus was allowed in the data processing, although our purpose in this study was not to find novel proteoforms, but to establish a novel approach for N-terminomics. Furthermore, to compare our results with two published N-terminome datasets for HEK293T human cells $(17,18)$, we re-analyzed those datasets under the same conditions without the use of their original customized database or non-specific cleavage. In terms of the contents of acetylated and unmodified protein N-terminal peptides, all three datasets provided 
identical results, whereas the content of internal peptides as well as the number of unique peptides varied depending on the approach and the sample amount (Supplementary Figure S4).

In conclusion, we have succeeded in developing a new N-terminomics method that does not require chemical reactions. This simple and rapid approach is suitable for highthroughput screening with minimal sample amounts. Our TrypN/CHAMP-based Nterminomics can enrich protein N-terminal peptides without bias, including peptides containing basic amino acids, with or without $\mathrm{N}$-terminal modifications. We believe CHAMP-SCX has great potential for expanding N-terminomics. Potential developments include deeper profiling with additional fractionation, the use of customized databases containing predicted protein N-termini, the replacement of HPLC with StageTips for CHAMP separation, and quantification by isotopic labeling.

\section{ACKNOWLEDGMENTS}

We would like to thank members of Department of Molecular \& Cellular BioAnalysis for fruitful discussions. This work was supported by JST Strategic Basic Research Program CREST (No. 18070870) and JSPS Grant-in-Aid for Scientific Research No. 17 H05667.

\section{DATA AVAILABILITY}

All LC/MS/MS data that support the findings of this study have been deposited with the ProteomeXchange Consortium via the jPOST partner repository with the dataset identifier JPST000422 (PXD010551) (34). 


\section{REFERENCES}

1. Nakahigashi, K., Takai, Y., Kimura, M., Abe, N., Nakayashiki, T., Shiwa, Y., Yoshikawa, H., Wanner, B. L., Ishihama, Y., and Mori, H. (2016) Comprehensive identification of translation start sites by tetracycline-inhibited ribosome profiling. DNA Res. 23, 193-201

2. Ingolia, N. T. (2014) Ribosome profiling: new views of translation, from single codons to genome scale. Nat. Rev. Genet. 15, 205-213

3. Van Damme, P., Gawron, D., Van Criekinge, W., and Menschaert, G. (2014) N-terminal Proteomics and Ribosome Profiling Provide a Comprehensive View of the Alternative Translation Initiation Landscape in Mice and Men. Mol. Cell. Proteomics 13, 1245-1261

4. Hwang, C. S., Shemorry, A., and Varshavsky, A. (2010) N-Terminal Acetylation of Cellular Proteins Creates Specific Degradation Signals. Science 327, 973-977

5. Starheim, K. K., Gevaert, K., and Arnesen, T. (2012) Protein N-terminal acetyltransferases: when the start matters. Trends Biochem. Sci. 37, 152-161

6. Mahrus, S., Trinidad, J. C., Barkan, D. T., Sali, A., Burlingame, A. L., and Wells, J. A. (2008) Global sequencing of proteolytic cleavage sites in apoptosis by specific labeling of protein $\mathrm{N}$ termini. Cell 134, 866-876

7. McDonald, L., Robertson, D. H. L., Hurst, J. L., and Beynon, R. J. (2005) Positional proteomics: selective recovery and analysis of $\mathrm{N}$-terminal proteolytic peptides. Nat. Methods 2, 955-957

8. Leitner, A. (2018) A review of the role of chemical modification methods in contemporary mass spectrometry-based proteomics research. Anal. Chim. Acta 1000, 2-19 
9. Klein, T., Eckhard, U., Dufour, A., Solis, N., and Overall, C. M. (2018) Proteolytic Cleavage-Mechanisms, Function, and "Omic" Approaches for a Near-Ubiquitous Posttranslational Modification. Chem. Rev. 118, 1137-1168

10. Xu, G., Shin, S. B., and Jaffrey, S. R. (2009) Global profiling of protease cleavage sites by chemoselective labeling of protein N-termini. Proc. Natl Acad. Sci. U.S.A. 106, 19310-19315

11. Varland, S., Osberg, C., and Arnesen, T. (2015) N-terminal modifications of cellular proteins: The enzymes involved, their substrate specificities and biological effects. Proteomics 15, 2385-2401

12. Lai, Z. W., Petrera, A., and Schilling, O. (2015) Protein amino-terminal modifications and proteomic approaches for N-terminal profiling. Curr. Opin. Chem. Biol. 24, 71-79

13. Gevaert, K., Goethals, M., Martens, L., Van Damme, J., Staes, A., Thomas, G. R., and Vandekerckhove, J. (2003) Exploring proteomes and analyzing protein processing by mass spectrometric identification of sorted N-terminal peptides. Nat. Biotechnol. 21, 566-569

14. Kleifeld, O., Doucet, A., auf dem Keller, U., Prudova, A., Schilling, O., Kainthan, R. K., Starr, A. E., Foster, L. J., Kizhakkedathu, J. N., and Overall, C. M. (2010) Isotopic labeling of terminal amines in complex samples identifies protein $\mathrm{N}$-termini and protease cleavage products. Nat. Biotechnol. 28, 281-288

15. Venne, A. S., Solari, F. A., Faden, F., Paretti, T., Dissmeyer, N., and Zahedi, R. P. (2015) An improved workflow for quantitative N-terminal charge-based fractional diagonal chromatography (ChaFRADIC) to study proteolytic events in Arabidopsis thaliana. Proteomics 15, 2458-2469 
16. Chen, L. F., Shan, Y. C., Weng, Y. J., Sui, Z. G., Zhang, X. D., Liang, Z., Zhang, L. H., and Zhang, Y. K. (2016) Hydrophobic Tagging-Assisted N-Termini Enrichment for InDepth N-Terminome Analysis. Anal. Chem. 88, 8390-8395

17. Na, C. H., Barbhuiya, M. A., Kim, M. S., Verbruggen, S., Eacker, S. M., Pletnikova, O., Troncoso, J. C., Halushka, M. K., Menschaert, G., Overall, C. M., and Pandey, A. (2018) Discovery of noncanonical translation initiation sites through mass spectrometric analysis of protein N termini. Genome Res. 28, 25-36

18. Yeom, J., Ju, S., Choi, Y., Paek, E., and Lee, C. (2017) Comprehensive analysis of human protein N-termini enables assessment of various protein forms. Sci. Rep. 7, 6599

19. Adachi, J., Hashiguchi, K., Nagano, M., Sato, M., Sato, A., Fukamizu, K., Ishihama, Y., and Tomonaga, T. (2016) Improved Proteome and Phosphoproteome Analysis on a Cation Exchanger by a Combined Acid and Salt Gradient. Anal. Chem. 88, 7899-7903

20. Essader, A. S., Cargile, B. J., Bundy, J. L., and Stephenson, J. L., Jr. (2005) A comparison of immobilized $\mathrm{pH}$ gradient isoelectric focusing and strong-cation-exchange chromatography as a first dimension in shotgun proteomics. Proteomics 5, 24-34

21. Helbig, A. O., Gauci, S., Raijmakers, R., van Breukelen, B., Slijper, M., Mohammed, S., and Heck, A. J. R. (2010) Profiling of N-Acetylated Protein Termini Provides In-depth Insights into the N-terminal Nature of the Proteome. Mol. Cell. Proteomics 9, 928-939

22. Gauci, S., Helbig, A. O., Slijper, M., Krijgsveld, J., Heck, A. J., and Mohammed, S. (2009) Lys-N and trypsin cover complementary parts of the phosphoproteome in a refined SCXbased approach. Anal. Chem. 81, 4493-4501

23. Wilson, J. P., Ipsaro, J. J., Del Giudice, S. N., Turna, N. S., Gauss, C. M., Dusenbury, K. H., Marquart, K., Rivera, K. D., and Pappin, D. J. (2020) Tryp-N: A Thermostable 
Protease for the Production of N-terminal Argininyl and Lysinyl Peptides. J. Proteome Res. 19, 1459-1469

24. Tallant, C., Garcia-Castellanos, R., Seco, J., Baumann, U., and Gomis-Ruth, F. X. (2006) Molecular analysis of ulilysin, the structural prototype of a new family of metzincin metalloproteases. J. Biol. Chem. 281, 17920-17928

25. Huesgen, P. F., Lange, P. F., Rogers, L. D., Solis, N., Eckhard, U., Kleifeld, O., Goulas, T., Gomis-Ruth, F. X., and Overall, C. M. (2015) LysargiNase mirrors trypsin for protein C-terminal and methylation-site identification. Nat. Methods 12, 55-58

26. Koneru, L., Ksiazek, M., Waligorska, I., Straczek, A., Lukasik, M., Madej, M., Thogersen, I. B., Enghild, J. J., and Potempa, J. (2016) Mirolysin, a LysargiNase from Tannerella forsythia, proteolytically inactivates the human cathelicidin, LL-37. Biol. Chem. 398, 395-409.

27. Tsiatsiani, L., Giansanti, P., Scheltema, R. A., van den Toorn, H., Overall, C. M., Altelaar, A. F. M., and Heck, A. J. R. (2017) Opposite Electron-Transfer Dissociation and HigherEnergy Collisional Dissociation Fragmentation Characteristics of Proteolytic K/R(X)(n) and $(\mathrm{X})(\mathrm{n}) \mathrm{K} / \mathrm{R}$ Peptides Provide Benefits for Peptide Sequencing in Proteomics and Phosphoproteomics. J. Proteome Res. 16, 852-861

28. Masuda, T., Tomita, M., and Ishihama, Y. (2008) Phase transfer surfactant-aided trypsin digestion for membrane proteome analysis. J. Proteome Res. 7, 731-740

29. Rappsilber, J., Ishihama, Y., and Mann, M. (2003) Stop and go extraction tips for matrixassisted laser desorption/ionization, nanoelectrospray, and LC/MS sample pretreatment in proteomics. Anal. Chem. 75, 663-670 
30. Tyanova, S., Temu, T., and Cox, J. (2016) The MaxQuant computational platform for mass spectrometry-based shotgun proteomics. Nat. Protocols 11, 2301-2319

31. Riley, M., Abe, T., Arnaud, M. B., Berlyn, M. K. B., Blattner, F. R., Chaudhuri, R. R., Glasner, J. D., Horiuchi, T., Keseler, I. M., Kosuge, T., Mori, H., Perna, N. T., Plunkett, G., Rudd, K. E., Serres, M. H., Thomas, G. H., Thomson, N. R., Wishart, D., and Wanner, B. L. (2006) Escherichia coli K-12: a cooperatively developed annotation snapshot 2005. Nucleic Acids Res. 34, 1-9

32. Raijmakers, R., Neerincx, P., Mohammed, S., and Heck, A. J. (2010) Cleavage specificities of the brother and sister proteases Lys-C and Lys-N. Chem. Commun. (Camb) 46, 8827-8829

33. Gussakovsky, D., Neustaeter, H., Spicer, V., and Krokhin, O. V. (2017) SequenceSpecific Model for Peptide Retention Time Prediction in Strong Cation Exchange Chromatography. Anal. Chem. 89, 11795-11802

34. Okuda, S., Watanabe, Y., Moriya, Y., Kawano, S., Yamamoto, T., Matsumoto, M., Takami, T., Kobayashi, D., Araki, N., Yoshizawa, A. C., Tabata, T., Sugiyama, N., Goto, S., and Ishihama, Y. (2017) jPOSTrepo: an international standard data repository for proteomes. Nucleic Acids Res. 45, D1107-D1111

\section{FOOTNOTES}

${ }^{*}$ Corresponding Author: Dr. Yasushi Ishihama

Tel: +81-75-753-4555 Fax: +81-75-753-4601

E-mail: yishiham@pharm.kyoto-u.ac.jp 


\section{TABLES}

Table 1. Enrichment of $E$. coli protein N-terminal peptides by SCX HPLC with isocratic elution at three different $\mathrm{KCl}$ concentrations.

\begin{tabular}{lccc}
\hline \multicolumn{1}{c}{ Salt concentration } & $\mathbf{1 0} \mathbf{~ m M}$ & $\mathbf{1 2 . 5} \mathbf{~ m}$ & $\mathbf{1 5} \mathbf{~ M}$ \\
\hline $\begin{array}{l}\text { Unique peptides } \\
\text { Unmodified protein N-terminal }\end{array}$ & 432 & 1,669 & 3,416 \\
$\begin{array}{l}\text { peptides } \\
\text { Acetylated protein N-terminal }\end{array}$ & 326 & 444 & 387 \\
$\begin{array}{l}\text { peptides } \\
\text { Protein N-terminal peptides (\%) }\end{array}$ & 31 & 25 & 22 \\
$\begin{array}{l}\text { based on peptide counts } \\
\text { Protein N-terminal peptides (\%) }\end{array}$ & 82.6 & 28.1 & 12.0 \\
based on peak area & 98.2 & 49.2 & 18.5 \\
\hline
\end{tabular}

The enrichment specificity of protein N-terminal peptides is obtained by calculating the number and LC/MS peak area of protein N-terminal peptides among all identified peptides. 
Table 2. Identification of protein N-terminal peptides from TrypN-digested HEK293T

cells.

\begin{tabular}{lcccc}
\hline & Replicate 1 & Replicate 2 & Replicate 3 & Total \\
\hline $\begin{array}{l}\text { Unmodified protein N-terminal } \\
\text { peptides }\end{array}$ & $199( \pm 3)$ & $187( \pm 5)$ & $197( \pm 3)$ & 352 \\
$\begin{array}{l}\text { Acetylated protein N-terminal } \\
\text { peptides }\end{array}$ & $1,854( \pm 13)$ & $1,301( \pm 18)$ & $1,509( \pm 15)$ & 2,666 \\
$\begin{array}{l}\text { Internal peptides } \\
\text { N-term ratio (\%, peptide }\end{array}$ & $160( \pm 8)$ & $147( \pm 3)$ & $232( \pm 6)$ & 433 \\
counts) & $92.8( \pm 0.3)$ & $91.0( \pm 0.2)$ & $88.0( \pm 0.3)$ & \\
N-term ratio (\%, peptide area) & $97.4( \pm 0.3)$ & $98.0( \pm 0.5)$ & $97.4( \pm 0.5)$ & 167 \\
\hline $\begin{array}{l}\text { Unmodified protein groups } \\
\text { Partially acetylated protein } \\
\text { groups }\end{array}$ & $115( \pm 3)$ & $100( \pm 4)$ & $116( \pm 3)$ & 1,640 \\
$\begin{array}{l}\text { Acetylated protein groups } \\
\text { (36) }\end{array}$ & $1,223( \pm 7)$ & $1,000( \pm 9)$ & $1,187( \pm 16)$ \\
\hline
\end{tabular}

Samples were prepared in triplicate (Replicates 1-3) and nanoLC/MS/MS of each sample was conducted in triplicate. Each number in the table is the average of triplicate measurements, and the total number is calculated after merging all results $(n=9)$ and removing redundancy. The enrichment specificity of protein $\mathrm{N}$-terminal peptides is obtained by calculating the number and peak area of protein $\mathrm{N}$-terminal peptides among all identified peptides. 


\section{FIGURES AND FIGURE LEGENDS}
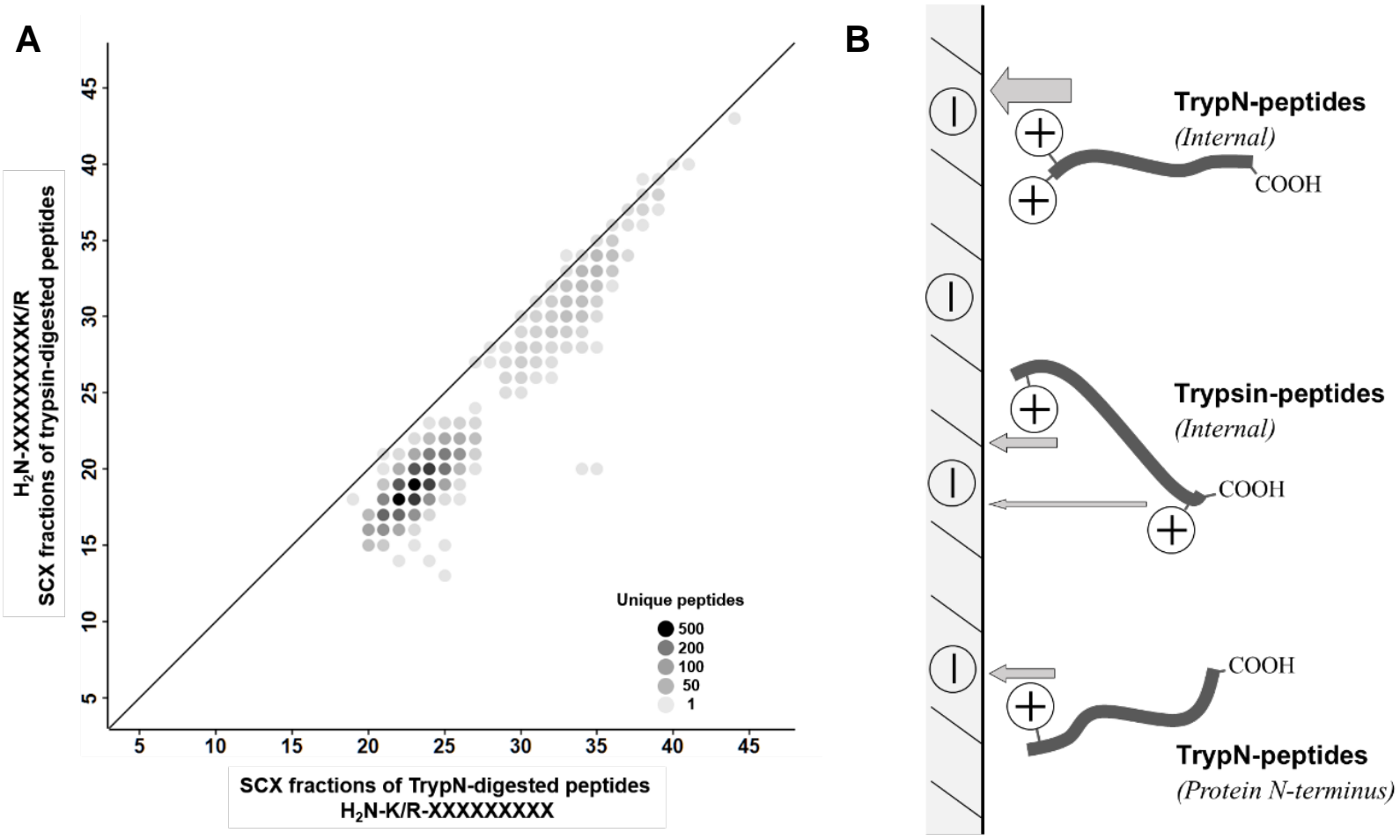

Figure 1. SCX separation of TrypN-digested and trypsin-digested peptides.

(A) Comparison of SCX elution profiles of TrypN- and trypsin-digested peptides. Peptides with the same sequence except for the termini were selected (K/R-XXXXXX and XXXXXX-K/R for TrypN- and trypsin-digested peptides, respectively). The shade of the circle color indicates the number of peptides.

(B) Proposed retention model of TrypN-digested internal peptides, trypsin-digested internal peptides and TrypN-digested protein N-terminal peptides on SCX stationary phase. 


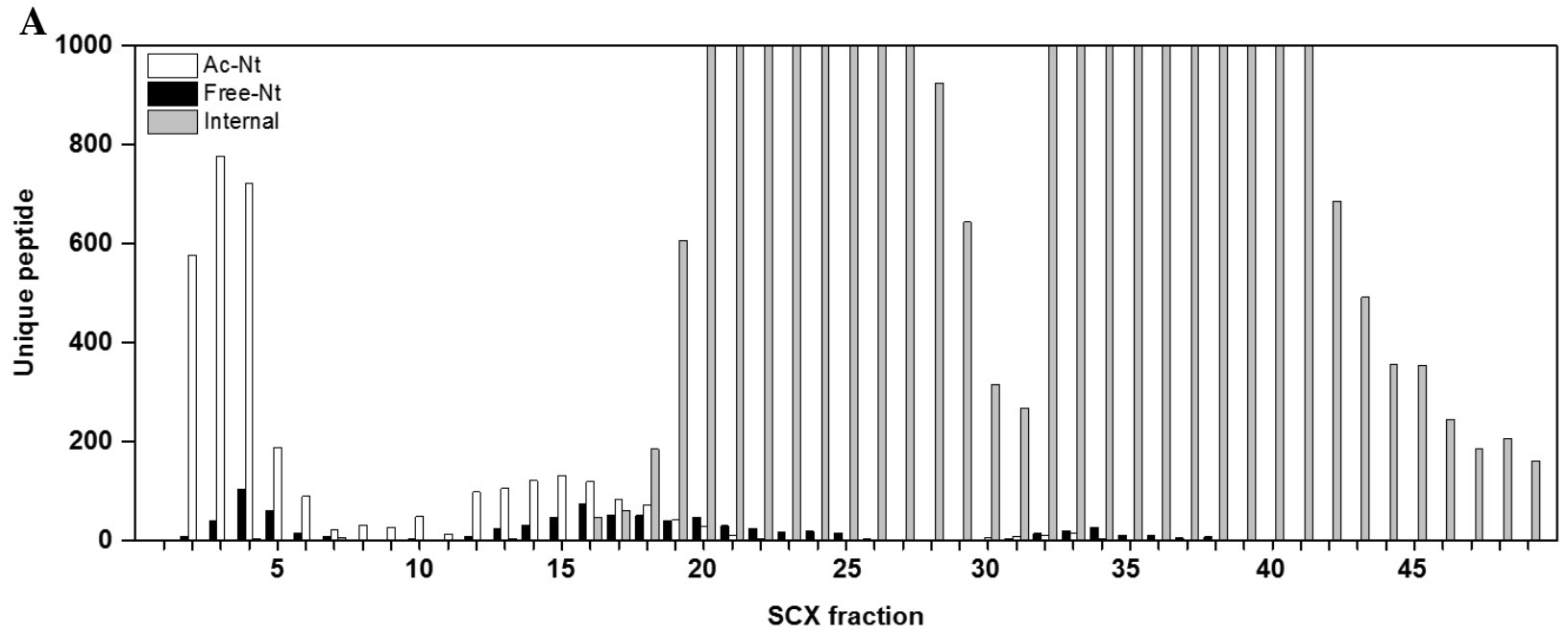

B

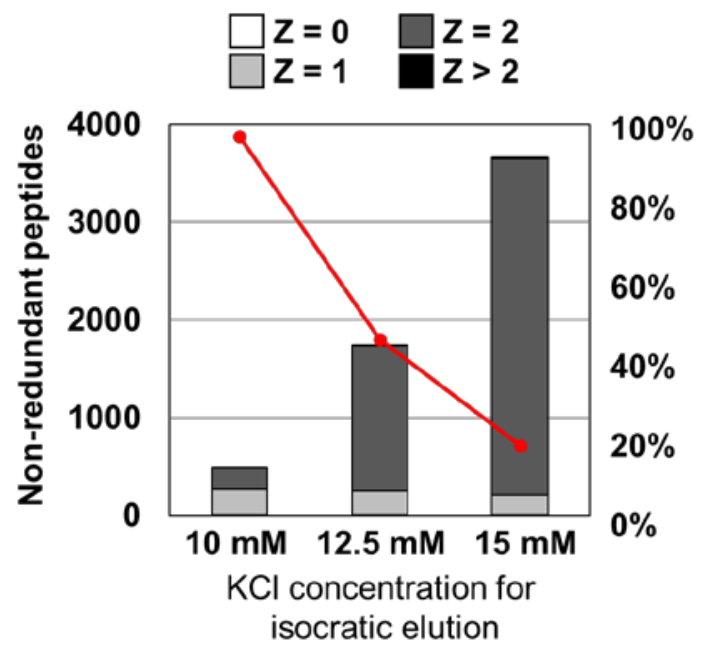

C

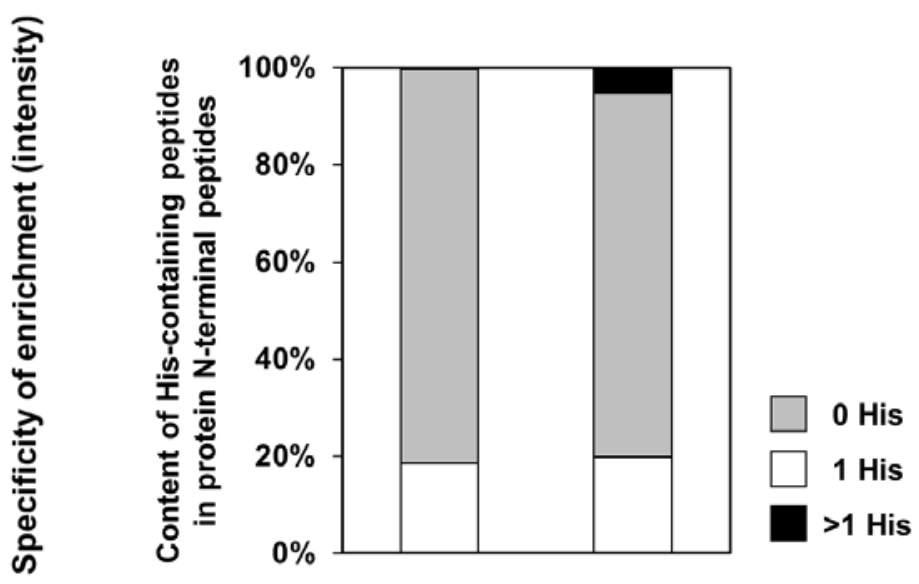

Figure 2. SCX elution profiles of TrypN-digested peptides.

(A) SCX HPLC fractionation of different types of peptides in TrypN-digested HEK293T cell lysates using $\mathrm{KCl}$ salt gradient elution. In fractions 2-6, protein $\mathrm{N}$-terminal peptides with $\mathrm{Z}=0$ and 1 ( $\mathrm{Z}$ is the total of unmodified $\mathrm{N}$-terminus and the number of basic amino acid residues) were observed, such as acetylated protein N-terminal peptides with or without a basic amino acid residue and unmodified protein Nterminal peptides without a basic amino acid residue. 
(B) SCX HPLC separation of TrypN-digested E. coli peptides under isocratic conditions with different $\mathrm{KCl}$ concentrations. The specificity in enriching protein N-terminal peptides and the numbers of peptides with different $\mathrm{Z}$ values are shown.

(C) The content of His-containing peptides in protein N-terminal peptides. The experimental results were those obtained with $10 \mathrm{mM} \mathrm{KCl}$ isocratic elution, and the in-silico results were calculated from the E. coli K-12 MG1665 protein sequence database. Details of in-silico digestion are described in the supplemental methods. 
A

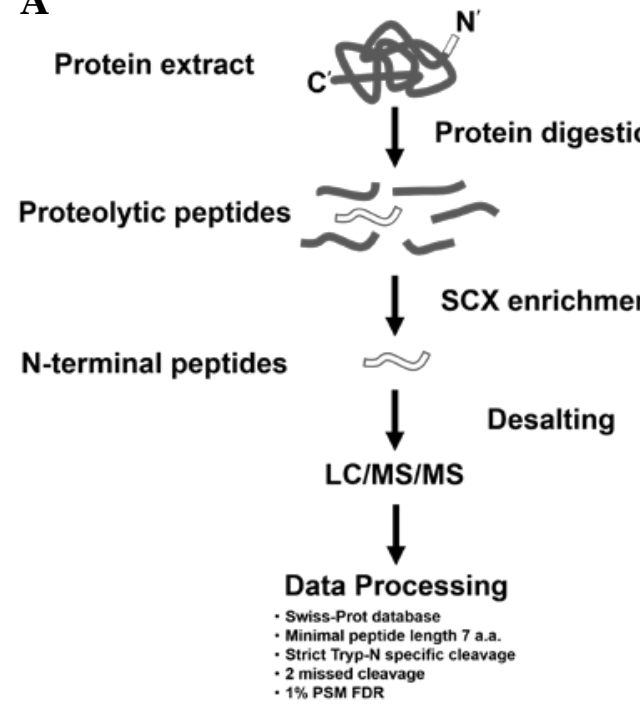

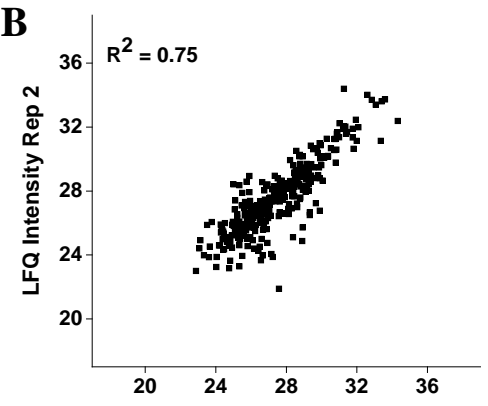


C

- Internal peptides

$\square$ N-terminal peptides

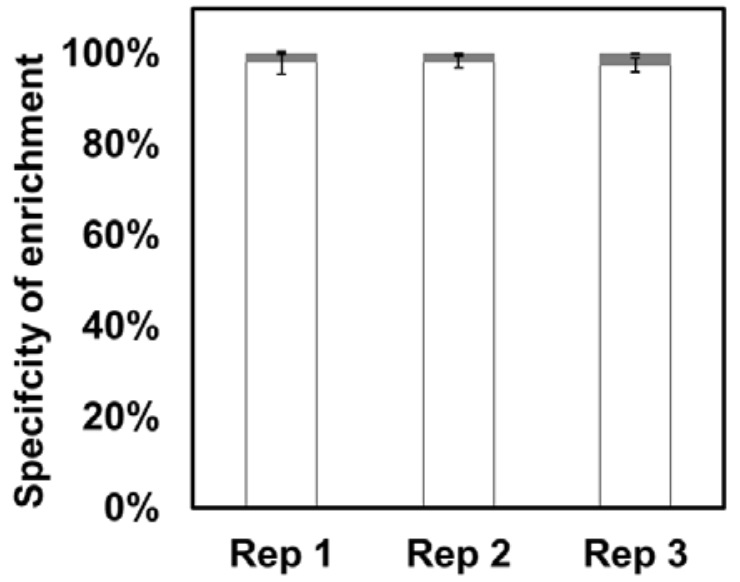

Figure 3. N-Terminal proteomics using CHAMP-SCX.

(A) Workflow of TrypN/CHAMP-based N-terminal proteomics. For details, see Materials and Methods. Three technical replicates were conducted on Day 1 (Rep 1) and Day 2 (Rep 2 and Rep 3) to evaluate inter- and intraday reproducibility.

(B) Reproducibility in quantifying peptide peak areas between three technical replicates.

(C) Enrichment specificity for protein N-terminal peptides in three repeats. 
bioRxiv preprint doi: https://doi.org/10.1101/2020.06.15.153684; this version posted June 16, 2020. The copyright holder for this preprint (which was not certified by peer review) is the author/funder, who has granted bioRxiv a license to display the preprint in perpetuity. It is made available under aCC-BY-NC-ND 4.0 International license. 